\title{
Scorpionates: Coordination Chemistry Comes Home
}

\author{
Roman Kresinski, Seema S. Pillai, Peter J S Foot \\ School of Materials Engineering, Kingston University, United Kingdom, Kingston-upon-Thames, Penrhyn Road, \\ r.kresinski@kingston.ac.uk
}

Abstract - Some underreported aspects of polypyrazolylborate chemistry are explored, in particular novel lanthanide coordination chemistry and zwitterionic ligand replacements of this so-called "scorpionate" class of ligands. The application of these ligands to future materials engineering is postulated and, finally, the historic relevance of the topic to $L$ 'viv is described.

Keywords - scorpionates, scorpionate ligands, polypyrazolylborates, polypyrazolylborate ligands, Trofimenko, Swiatoslaw, materials engineering, lanthanide ions.

\section{Introduction}

Since their reporting [1] by Swiatoslaw Trofimenko in 1967 the polypyrazolylborate, or 'scorpionate', class of ligands ( $\mathrm{Tp}^{-}$, Figure 1) has been used to form complexes with a wide variety of metals around the periodic table [2].

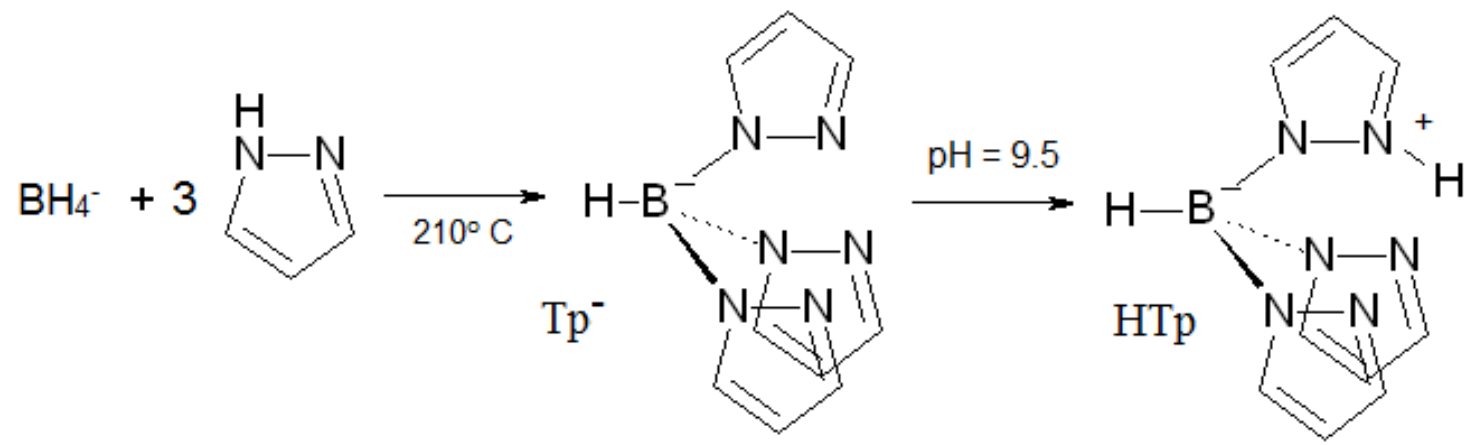

Figure 1: Synthesis of Tp- and HTp

The trivalent lanthanide metal ions possess unique and desirable properties such as high magnetism or luminescence which might be incorporated into polymeric 'plastics' to afford cheaply shaped, but highly specialised, materials. We were interested in the idea of fixing one lanthanide ion proximally to one double bond in the hope that the resulting materials might be amenable to reproducible copolymerisation to afford a reasonably homogeneous copolymer, locating Ln centres at predictably-distant 'island' points in any product, wherefrom they might migrate only under great stress. Our interest in scorpionate chemistry is reinforced by the observation that a stable family of lanthanide acetate complexes [ $\left.\mathrm{LnTp}_{2}\left(\mathrm{OOCCH}_{3}\right)\right]$ can be produced for the whole of the lanthanide series $(\mathrm{Ln}=\mathrm{La}-\mathrm{Lu})$ [3]. Our hope is that the substitution of the acetate ligand for one containing $\mathrm{C}=\mathrm{C}$ double bonds might allow the incorporation of $\left\{\mathrm{LnTp}_{2}\right\}$ moieties, along with the desirable associated properties of Ln ions, into highly processible materials such as polymers, by co-polymerisation.

A second point of our interest in the scorpionate ligands is concerned with the synthesis of scorpionate complexes with d-block metals, immediately from other labile complexes, by direct use of the free acid HTp [1], Figure 1. This moderately stable and presumably zwitterionic species can act as a protonating agent for formally anionic ligands [4] and, concomitantly, as a chelating agent displacing existing neutral ligands from the metal coordination sphere. This under-explored class of reagents can afford, in labile complexes, the transformation of complexes containing one class of ligands, directly to those containing the Tp ligand. 


\section{Lanthanide Coordination Chemistry Studies}

Titration of a 2:1 mixture of aqueous $\mathrm{Tp}^{-}$: $\operatorname{crot}^{-}$into stirred aqueous solutions of lanthanide chlorides or nitrates afforded complexes of the expected [LnTp $(\operatorname{crot})] \quad\left(\operatorname{crot}^{-}=\right.$ $\mathrm{CH}_{3} \mathrm{CH}=\mathrm{CHCOO}^{-}$) formulation for the later lanthanides (Ho-Lu), confirmed by a single-crystal $\mathrm{X}$-ray study of the Ho complex (Figure 2).

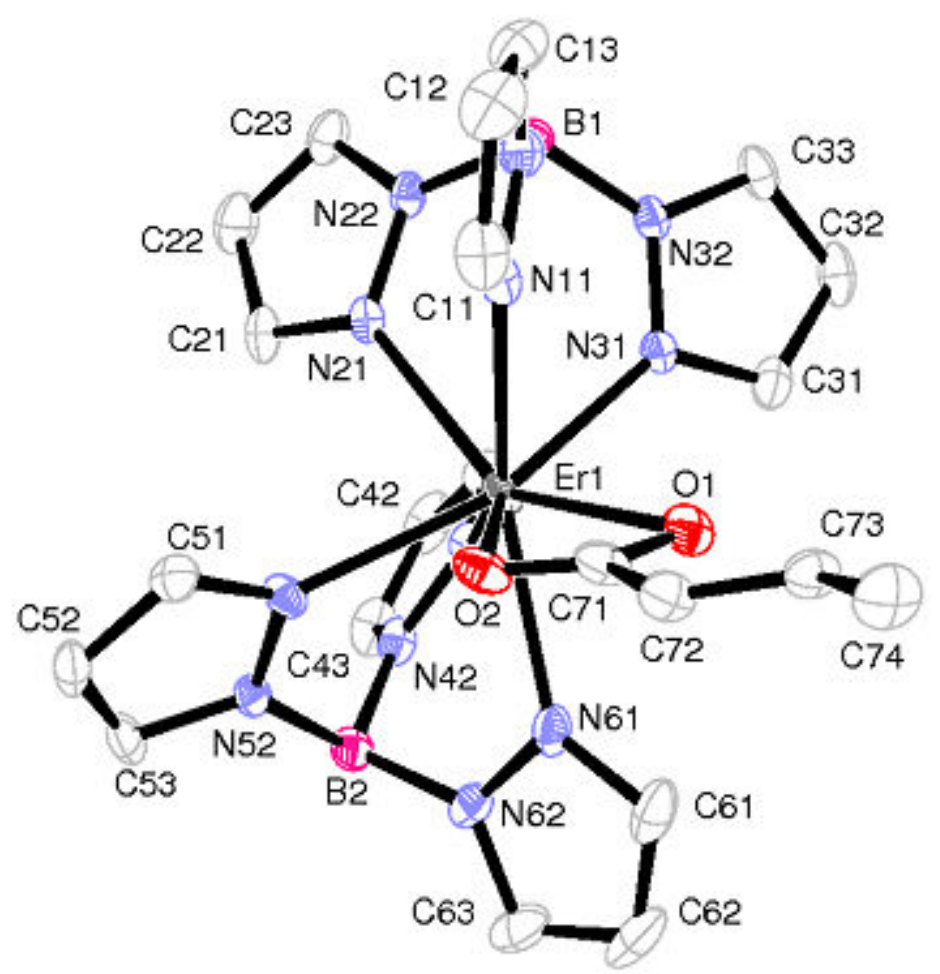

Figure 2. The single-crystal X-ray structure of [HoTp 2 (crot)]

However, it was clear from spectroscopic data that we had formed complexes falling into two morphological families, whose infrared properties are summarised in Table 1.

Table 1: Selected infrared spectral data for lanthanide crotonate complexes

\begin{tabular}{|c|c|c|c|c|c|}
\hline & $\mathrm{U}_{(\mathrm{BH})}$ & $\mathrm{v}_{(\mathrm{CO})}$ & & $\mathrm{U}_{(\mathrm{BH})}$ & $\mathrm{v}_{(\mathrm{CO})}$ \\
\hline $\mathrm{La}$ & - & - & $?\left[\mathrm{DyTp}(\operatorname{crot})_{2}\right]_{2}$ & $2453 / 2494$ & 1657 \\
\hline$\left[\mathrm{CeTp}(\mathrm{crot})_{2}\right]_{2}$ & 2451 & 1661 & {$\left[\operatorname{HoTp}(\operatorname{crot})_{2}\right]_{2}^{a}$} & 2494 & 1656 \\
\hline$\left[\operatorname{PrTp}(\operatorname{crot})_{2}\right]_{2}$ & 2452 & 1660 & {$\left[\mathrm{HoTp}_{2}(\mathrm{crot})\right]^{\mathrm{b}}$} & 2474 & 1655 \\
\hline$\left[\mathrm{NdTp}(\operatorname{crot})_{2}\right]_{2}$ & 2442 & 1660 & $?\left[\operatorname{ErTp}_{2}(\operatorname{crot})\right]$ & $2458 / 2447$ & 1665 \\
\hline$\left[\operatorname{SmTp}(\operatorname{crot})_{2}\right]_{2}$ & 2447 & 1657 & {$\left[\operatorname{TmTp}_{2}(\mathrm{crot})\right]$} & 2475 & 1655 \\
\hline$\left[\mathrm{EuTp}(\operatorname{crot})_{2}\right]_{2}$ & 2450 & 1657 & {$\left[\mathrm{YbTp}_{2}(\mathrm{crot})\right]$} & 2475 & 1655 \\
\hline$\left[\mathrm{GdTp}(\operatorname{crot})_{2}\right]_{2}$ & 2451 & 1658 & {$\left[\mathrm{LuTp}_{2}(\mathrm{crot})\right]$} & 2475 & 1656 \\
\hline$\left[\mathrm{TbTp}(\operatorname{crot})_{2}\right]_{2}$ & 2452 & 1659 & & & \\
\hline
\end{tabular}

The earlier, larger lanthanide ions adopt a novel structural type $\left[\operatorname{LnTp}(\mathrm{crot})_{2}\right]_{2}$ incorporating unusual $\left(\mu_{2}-\kappa^{2}, \kappa^{1}\right)$ bridging crotonate ligands, affording the metal ions 8coordination (Figure 3). 


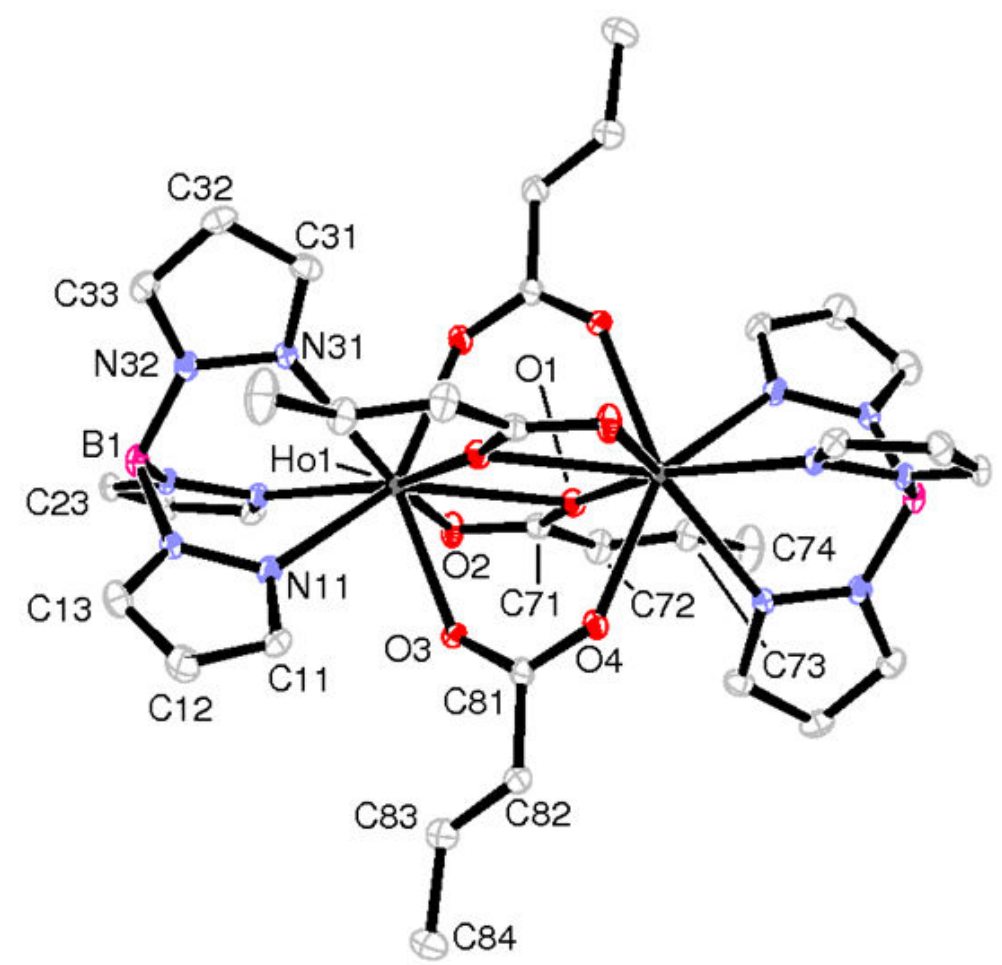

Figure 3. The single-crystal X-ray structure of $\left[\mathrm{HoTp}(\operatorname{crot})_{2}\right]_{2}$

Clearly either complex type might be potentially suitable for co-polymerisation, and we have also obtained potentially useful analogous cinnamate complexes for future study.

\section{Free Acid Studies}

The free acid HTp, like the free acids of all other polypyrazolylborates, is of only moderate stability, decomposing over a few days [1]. However, it does have a surprisingly low melting point of about $95{ }^{\circ} \mathrm{C}$, allowing it to be molten together with its potential co-reagent. In this way a variety of ligand replacement reactions may be carried out, as shown in Figure 4.

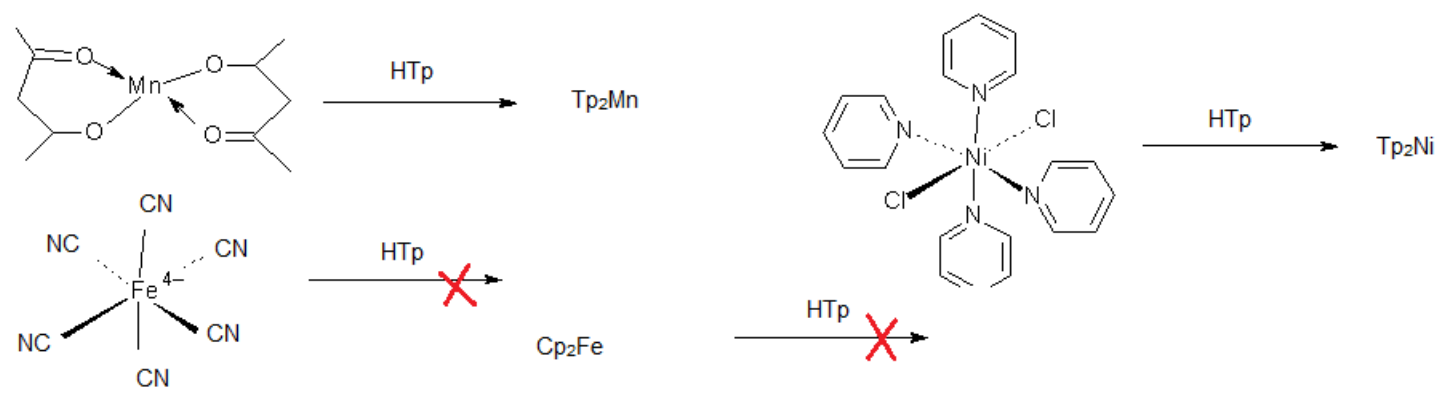

Figure 4. Typical reactions of the free acid HTp

The concept of the reactions proceeding by ligand protonation, followed by replacement of ligands by $\mathrm{Tp}$ seemed a straightforward one - for example the reaction $\mathrm{Cp}_{2} \mathrm{ZrCl}_{2}+\mathrm{HTp}-->$ $\mathrm{TpCpZrCl} 2+\mathrm{HCp}\left(\mathrm{Cp}^{-}=\mathrm{C}_{5} \mathrm{H}_{5}^{-}\right)$was accompanied by the aroma of cyclopentadiene. However, no amount of HTp, moderate or excess [5], when reacted with $\mathrm{Cp}_{2} \mathrm{ZrCl}_{2}$, would afford $\mathrm{Tp}_{2} \mathrm{ZrCl}_{2}$. Instead, ill-characterised $\mathrm{Zr}$ species, possibly $\mathrm{TpZrCl}_{3}$ derivatives, were isolated as well as 1,4bispyrazolylpyrazaboles (Figure 5), which are the breakdown products of HTp and whose relative proportions (1,4-cis- or 1,4-trans-) are dictated by the absence or presence of $\mathrm{Cp}_{2} \mathrm{ZrCl}_{2}$ [6]. 


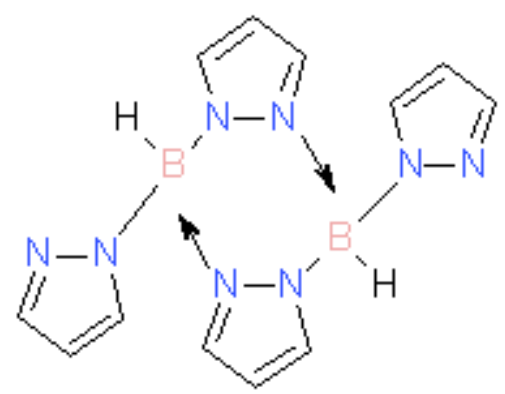

Figure 5. 1,4-bispyrazolylpyrazabole

\section{Conclusion}

The latter few sentences above conclude this brief account of polypyrazolylborate (or scorpionate) chemistry in an appropriate way, interrelating the three main advances introduced by Swiatoslaw Trofimenko in the mid-1960s. In two seminal papers in the mid-1960s he described the new polypyrazolylborate ligands [1], their novel free acids [1] and the new pyrazaboles [7]. The authors summarise only a few of their own observations, spanning four decades, interrelating the above original themes in novel ways. There is so much more.

Swiatoslaw 'Jerry' Trofimenko passed away in the USA on 26 February 2007, but he was born in Lviv in 1931. The authors are humbly grateful in 2019 to have the pleasure of describing some of their own investigations, of chemistry originating from Lviv, at the place of its 'origin'.

\section{Acknowledgments}

The authors wish to thank Prof. Jon McCleverty, Dr Chris Jones and Prof Peter Foot for past support. This short account is dedicated to the memory of Swiatoslaw Trofimenko.

\section{References}

[1] S. Trofimenko, "Boron-pyrazole chemistry. II. Poly(1-pyrazolyl)-borates" J. Am. Chem. Soc., 89, 13, pp. 3170-3177, 1967.

[2] S. Trofimenko, "Polypyrazolylborates: Scorpionates" J. Chem. Educ., 82, pp. 1715-1720, 2005.

[3] M. A. J. Moss and C. J. Jones, "Heteroleptic poly(pyrazolyl)borate derivatives of the lanthanide ions. The synthesis of tropolonate and carboxylate complexes and the $X$-ray crystal structures of $\left[\mathrm{Yb}\left\{\mathrm{HB}\left(\mathrm{C}_{3} \mathrm{~N}_{2} \mathrm{H}_{3}\right)_{3}\right\}_{2}\left(\mathrm{O}_{2} \mathrm{C}_{7} \mathrm{H}_{5}\right)\right]$ and $\left[\mathrm{Yb}\left\{\mathrm{HB}\left(\mathrm{C}_{3} \mathrm{~N}_{2} \mathrm{H}_{3}\right)_{3}\right\}_{2}\left(\mathrm{O}_{2} \mathrm{CPh}\right)\right]$ " J. Chem. Soc. Dalton Trans., pp. 581-591, 1990.

[4] R. A. Kresinski, C. J. Jones and J. A. McCleverty, "Cyclopentadienyl replacement by trispyrazolylborate and the syntheses of $\mathrm{M}\left(\eta^{5}-\mathrm{C}_{5} \mathrm{H}_{5}\right)\left\{\mathrm{HB}\left(\mathrm{C}_{3} \mathrm{H}_{3} \mathrm{~N}_{2}\right)_{3}\right\} \mathrm{X}_{2}(\mathrm{M}=\mathrm{Hf}, \mathrm{X}=\mathrm{Cl}$; $\left.\mathrm{M}=\mathrm{Zr}, \mathrm{X}=\mathrm{Cl}, \mathrm{OPh}, \mathrm{OC}_{6} \mathrm{H}_{4}-4-\mathrm{NO}_{2}, \mathrm{OC}_{6} \mathrm{H}_{4}-4-\mathrm{OMe}, \mathrm{OC}_{6} \mathrm{H}_{4}-2-\mathrm{Ph}\right)$ ]" Polyhedron, 9, 17, pp. 2185-2187, 1990.

[5] R A Kresinski, T. A. Hamor, C. J. Jones and J A McCleverty, "Syntheses of some cyclopentadienyl[hydrotris(pyrazol-1-yl)borato] bis(aryloxo)zirconium(IV) complexes and the X-ray crystal structure of $\left[\mathrm{Zr}\left(\eta^{5}-\mathrm{C}_{5} \mathrm{H}_{5}\right)\left\{\kappa^{3}-\mathrm{HB}(\mathrm{pz})_{3}\right\}\left(\mathrm{OC}_{6} \mathrm{H}_{4} \mathrm{Ph}-2\right)_{2}\right] "$ J. Chem. Soc. Dalton Trans., 603-607, 1990.

[6] R. A. Kresinski, "Pyrazabole formation by thermolysis of the free acid of the hydrotris(pyrazolyl)borate ion", J. Chem. Soc. Dalton Trans., pp. 401-405., 1999.

[7] S. Trofimenko, "Boron-pyrazole chemistry" J. Am. Chem. Soc., 88, 8, pp. 1842-1844, 1966. 